\title{
Grand challenges in theoretical and philosophical psychology: after psychology?
}

\section{Dan Lloyd*}

Department of Philosophy, Program in Neuroscience, Trinity College, Hartford, CT, USA

${ }^{*}$ Correspondence: dan.lloyd@trincoll.edu

Psychology is the rollercoaster of the sciences. In its brief history, psychology has swung through many -isms, alternately embracing and rejecting the widest variety of assumptions and first principles. In the decades ahead, psychology is once again facing metamorphoses, in its tectonic collision with neuroscience. This changing landscape is of profound significance for psychology, transforming the very idea of the discipline as well as providing new opportunities and challenges for many of its historical and contemporary offshoots and subfields.

In one sentence, the grand challenge facing psychology is simply this: What will the discipline of psychology be in the year 2020, in 2030, and beyond? (These decades are not so far away.)

Accordingly, the first challenge is definitional: What is psychology? Setting aside the dictionary definitions and their academic reifications, what at this point is the proper topic of psychology as a science? Is subsumption into the neurosciences the appropriate terminus, or will psychology stake out an autonomous domain of hypotheses and theories? If the latter, what will be the metaphysical and methodological commitments of the autonomous discipline, distinct from both brain and the antiquated soul? This existential question has several variants:

- In clinical psychology, what will remain of the concepts of mental health and illness? How will the constructs of self and other, choice and will, and the other core ideas of mental integrity, play out in the years ahead? How should the classification schemes of clinical psychology evolve? Patients and clinicians both appeal to folk psychological constructs in describing the experiences of healthy and ill individuals. How should folk-inflected concepts (like pain, depression, happiness, etc.) change?

- In cognitive psychology, what will "cognitive" denote? Computation served as model and metaphor for half a century. Will computation comfortably settle into the mold of neural networks? Will neural network models find a useful and informative middle ground between biological realism and loosely constrained abstraction?

- In developmental psychology, how does an emerging understanding of the developing brain affect models and theories of psychological growth? How does the genomic revolution reconfigure the relationship of innate and environmental influences in development? Since the brain never stops changing, how will concepts of development find application at every stage in human life?

- In social psychology, how will neuroscience (as theory and as folk metaphor) reconstitute the social world? Will technologies like "brain reading" and cloud computing transform human relationships and institutions?

- In comparative psychology, the decades ahead will offer many opportunities to consider non-Western conceptions as alternative frameworks for a neuroscientifically informed psychology. Will alternative conceptions of health and illness, modes of mental activity, relations of mind and body, etc., emerge as more appropriate descriptors in psychology?

In addition to the biological turn in the classic subdisciplines of psychology, in recent years several new theoretical directions have emerged, not necessarily tied to the upwelling of neuroscience. These have enriched the field, but are neither static nor outmoded. These overlapping and interacting themes should also be considered in their millennial form:

- Phenomenology: The project of "naturalizing phenomenology" offers the prospect of new explananda and sometimes new strategies of explanation in psychology. What methods and issues from phenomenology might inform a new psychology in the golden age of brain science?

- Embodiment: From several directions in recent years, driven by both philosophy and empirical science, the physical and bodily setting for behavior has emerged as a crucial constraint on any model of psychology. How does embodiment revise the concepts and methods available to a scientific psychology?

- Ecological Psychology: Just as psychology depends on the body, both brain and body exist in a rich actual environment; the perceptual psychology of Gibson and his followers challenge every theory in perception to accommodate to "ecologically valid" settings. Can this research program illuminate issues and ideas in new ways in this era of cognitive neuroscience?

- Evolutionary Psychology: The ultimate context for psychology is evolutionary; describing and explaining behavior as adaptation might reorganize the discipline of psychology, and root it in genetics. Will evolutionary psychology ultimately conform to the frameworks of other branches of psychology? How will the bridge from gene to brain to behavior ultimately be constructed?

Psychology is changing with these new influences and confluences. So also are several cognate disciplines, each of which faces its own challenges of definition and direction. These cognate disciplines can also cross-fertilize psychology, and cognate reconfigurations should be considered in their broadest psychological implications:

- Cognitive literary studies: The act of writing and reading, refracted through the genres of poetry, narrative, etc., and further refracted through the practices of diverse times and cultures, has become the theme of a lively new 
discipline in itself. Does this approach to literature point to new issues and concepts for psychology overall?

- Cognitive musicology: Like story-telling, music making seems to be universal in the human community (and rare among other species). Music might reflect basic functions of mind and brain; can the cognitive science and neuroscience of music illuminate broader issues in psychology as well?

- Cognitive studies of religious belief: Religious belief and practice cut across psychology with examples of behavior and cognition that defy ordinary rules of logic and practical reasoning. In their exceptionality, can religious behaviors reshape ideas about psychological processes in "rational" animals?

Transformations in its subdisciplines and movements always implicate the basic research methods and methodologies of scientific psychology, indicating another dimension of challenge and opportunity.

- Questions of method in contemporary psychology have been exponentiated by stunning advances in technology and technique. What are the implications of increasingly powerful measuring devices, their intricate observations, and the accompanying computational speed and capacity? Are there new methods that should be tested against the issues above? What is the appropriate relationship between hypothesis confirmation and data exploration in a data rich world?
- New methods in other disciplines will also affect psychology. Most conspicuously, the genetic contribution to behavior promises to loom large in the years ahead. Will psychology find a complete grounding in genomics? Is this a suitable goal for a scientific psychology? As genetics progresses, how should classic debates on the innate and the learned be revised as the etiology from gene to brain to behavior ramifies? At a more general level, how will reductionism transform the concepts and explanatory strategies that might be deployed? Are reductionism and eliminativism necessary partners?

Finally, just as neuroscience has begun a crossing from pure to applied science, with implications for ethics, all of the potential transformations questioned above can alter the place of psychology in society. The emerging field of neuroethics will have its psychological cognates. Put briefly, who owns the sciences of mind? Should research subjects, both patients and volunteers, become involved in research as more than just its object? In a world where policy is increasingly driven by science (or ought to be), what is the obligation of scientists to inform and educate general audiences? How should non-scientific constituencies shape the direction of research?

- One important dimension of this question involves the trend toward open source publication. This has been welcomed in every discipline. How can its benefits be extended, and its limitations superseded? Are there trade-offs among speed and rigor, exploration and confirmation, speculation and proof? How can the entire process of knowledge creation be made more transparent?

There are journals already dedicated to each of the domains and issues above. The Frontiers journals operate at the frontiers of all of them, but, more important, a Frontier journal can cross frontiers. This is especially important in conceiving the challenges and opportunities at the frontiers of theoretical and philosophical psychology. In the decades ahead, the subdisciplines and theoretical outlooks in psychology will be illuminated by creative borrowings of questions and methods across disciplinary lines. This network of influence implicates the natural sciences, social sciences, and humanities in the future of psychology. The grand challenge is to seize this grand opportunity.

Received: 22 March 2010; accepted: 24 March 2010; published online: 16 April 2010.

Citation: Lloyd D (2010) Grand challenges in theoretical and philosophical psychology: after psychology? Front. Psychology 1:9. doi: 10.3389/fpsyg.2010.00009

This article was submitted to Frontiers in Theoretical and Philosophical Psychology, a specialty of Frontiers in Psychology.

Copyright $\odot 2010$ Lloyd. This is an open-access article subject to an exclusive license agreement between the authors and the Frontiers Research Foundation, which permits unrestricted use, distribution, and reproduction in any medium, provided the original authors and source are credited. 